\title{
Translation and cross-cultural adaptation into Brazilian Portuguese of the Mood and Feelings Questionnaire (MFQ) - Long Version
}

\author{
Tradução e adaptação transcultural para o português brasileiro do instrumento \\ Mood and Feelings Questionnaire (MFQ) - Versão Longa
}

Martha Rosa, Elena Metcalf, Thiago Botter-Maio Rocha, Christian Kieling

\begin{abstract}
Introduction: Major depressive disorder (MDD) is prevalent among young people, with a high incidence during adolescence. It is, therefore, important to have reliable instruments to capture the construct of depression in this population. The objective of the present work is to describe the process of translation and cultural adaptation of the Mood and Feelings Questionnaire (MFQ) - Long Version, into Brazilian Portuguese.

Method: We followed the International Society for Pharmacoeconomics and Outcomes Research (ISPOR) guidelines for translation and cultural adaptation, including the steps of preparation, forward translation, reconciliation, back-translation, back-translation review, harmonization, cognitive debriefing, review of cognitive debriefing results and finalization, proofreading and final report. Cognitive debriefing was conducted in a sample of adolescent patients and their respective caregivers at mental health clinics affiliated with the Brazilian public health system.

Results: Results suggest that the items were well understood and that the MFQ seems to be an appropriate instrument for use with Brazilian adolescents and caregivers.

Conclusions: The Brazilian Portuguese MFQ - Long Version constitutes an adequate tool for the assessment of depression among adolescents. Future studies are required to evaluate psychometric properties of the instrument.
\end{abstract}

Keywords: Translation, adaptation, adolescent, depression.
Resumo

Introdução: $O$ transtorno depressivo maior (TDM) é prevalente em jovens, com alta incidência durante a adolescência. Portanto, é importante que instrumentos confiáveis estejam disponíveis para avaliar o construto da depressão nessa população. O objetivo do presente trabalho é descrever o processo de tradução e adaptação cultural do Mood and Feelings Questionnaire (MFQ) - Versão Longa para o português brasileiro.

Método: Foram utilizadas as diretrizes da International Society for Pharmacoeconomics and Outcomes Research (ISPOR) para tradução e adaptação cultural, incluindo as etapas de preparação, tradução, reconciliação, retrotradução, revisão da retrotradução, harmonização, estudo piloto, revisão dos resultados do estudo piloto, revisão final e relato final. A etapa de estudo piloto foi conduzida em uma amostra de pacientes adolescentes e seus respectivos cuidadores em clínicas de saúde mental afiliadas ao Sistema Único de Saúde.

Resultados: Os resultados sugeriram que os itens foram bem compreendidos, e que o MFQ parece ser um instrumento apropriado para uso com adolescentes e cuidadores brasileiros. Conclusões: A versão traduzida para o português brasileiro do MFQ - Versão Longa constitui um instrumento adequado para a avaliação da depressão em adolescentes. Futuros estudos são necessários para avaliar as propriedades psicométricas da escala.

Descritores: Tradução, adaptação, adolescente, depressão.

\footnotetext{
Departamento de Psiquiatria e Medicina Legal, Faculdade de Medicina, Universidade Federal do Rio Grande do Sul (UFRGS), Porto Alegre, RS, Brazil. Submitted Feb 14 2017, accepted for publication Jun 082017.

Suggested citation: Rosa M, Metcalf E, Rocha TB, Kieling C. Translation and cross-cultural adaptation into Brazilian Portuguese of the Mood and Feelings Questionnaire (MFQ) - Long Version. Trends Psychiatry Psychother. 2018;40(1):72-78. http://dx.doi.org/10.1590/2237-6089-2017-0019
} 


\section{Introduction}

Major depressive disorder (MDD) is prevalent among young people and represents a major cause of disease burden in this age group. ${ }^{1}$ The impact imposed by MDD among youth contrasts with the limited availability of studies and resources in low- and middle-income countries, where most of children and adolescents live. ${ }^{2}$ For this reason, it is crucial to have good and reliable measures to evaluate depressive symptomatology and to support clinical diagnoses and treatments in such contexts.

A number of self-report instruments are currently available for the assessment of children and adolescents. ${ }^{3}$ The Beck Depression Inventory (BDI) and Center for Epidemiologic Studies Depression Scale (CESD) were developed to evaluate depressive symptoms in adults, but are also validated for and used with the adolescent population. ${ }^{4-6}$ Although these scales have proven to be reliable measures, none of them provides a parental version for depressive symptom assessment in the caregiver, a relevant factor in the analysis of reported depressive symptomatology for this age group. ${ }^{3-6}$ Another widely used scale in this age group is the Children's Depression Inventory (CDI). The CDI was adapted from the BDI for children and adolescents but, despite having versions for both parents and teachers, the instrument is not freely available, which hinders its use in low- and middle-income settings. ${ }^{7}$

The Mood and Feelings Questionnaire (MFQ) is one of the most widely used instruments to assess MDD in children/adolescents and has been shown to be a reliable and valid measure. ${ }^{8}$ The MFQ was initially developed as a tool to be used in epidemiological studies of MDD.9,10 It was planned to cover not only Diagnostic and Statistical Manual of Mental Disorders (DSM) criteria, but also clinically relevant additional symptoms, such as feelings of loneliness, not being loved or being ugly. The instrument has three versions that can capture different perspectives of the depressive phenomenology in the child/adolescent and parent/caregiver. The versions developed for children/adolescents (MFQ-C) and for adults (MFQ-A) in their full form have 33 items referring to the subject him/herself. Additionally, the MFQ has a parental version (MFQ-P), which makes the instrument even more relevant, as it also evaluates the children's/ adolescents' depressive symptoms from the parent's perspective. The parental version of the scale in its full form has 34 items. All three versions are self-report questionnaires, evaluating symptoms over the previous two weeks with three response options rated as true (score $=2)$, sometimes true $($ score $=1$ ) and not true (score $=0)$. In addition to the long version, the MFQ has also a short questionnaire (SMFQ) that includes only 13 of the original items. These shorter versions (SMFQ-C and SMFQ-P) have been already translated into Brazilian Portuguese. ${ }^{11}$ Although the short form appears to be good in screening protocols, the long form includes a greater number of symptoms and provides a more comprehensive description of depressive symptoms, and it was adopted as the primary outcome measure in the largest randomized clinical trial so far conducted for this condition. ${ }^{12}$

Considering the high prevalence and the burden imposed by MDD across the lifespan, it is important to have adequate tools to capture this construct among children and adolescents. The translation and cultural adaptation processes of an instrument require a rigorous methodology, with the ultimate goal of maintaining the accuracy of the original scale. For this reason, the objective of this study is to describe the process of translation and cultural adaptation of MFQ-C and MFQ-P into Brazilian Portuguese using the procedures proposed by the Translation and Cultural Adaptation Group of the International Society for Pharmacoeconomics and Outcomes Research (ISPOR). ${ }^{13}$

\section{Method}

We followed all ISPOR recommended procedures for the translation and cultural adaptation process for self-report measures, encompassing ten steps. In the first step (preparation), we asked for the authors' permission to use the instrument, invited them to be involved in the process, and recruited translators. In the second step (forward translation), two independent translations were performed (by M.R. and V.M-.C. the latter is listed in the Acknowledgments). In the third step (reconciliation), the forward translations were reconciled into a single forward translation (any discrepancies were discussed with T.B.M.R. and C.K.). In step four, a native speaker of English (E.M.) performed a back-translation of the instrument into the original language. In step five, a back-translation review was conducted to ensure the conceptual equivalence of the translation with the original instrument. The sixth step consisted of harmonization across different translations. In step seven (cognitive debriefing), the level of comprehensibility of the translation was assessed in a sample of adolescents. The sample group for this stage was composed of patients treated at mental health clinics at Hospital de Clínicas de Porto Alegre. Specifically, 14 adolescents and their 14 respective primary caregivers were selected to answer the questionnaires. We used a convenience sampling approach, recruiting two adolescents (one boy and one girl) per year of age 
(from 11 to 17). There was no restriction in terms of psychiatric diagnoses; the only exclusion criterion was clinical evidence of intellectual disability. In step eight (review of cognitive debriefing results and finalization), results were reviewed and the translation was finalized. In step nine (proofreading), an additional revision was performed to check for possible errors that might have been missed during the translation process. The last, tenth step was the elaboration of this final report, detailing the translation process. This project was approved by the research ethics committee of Hospital de Clínicas de Porto Alegre (50473015.9.0000.5327).

\section{Results}

The process of translation and cultural adaptation generated Brazilian Portuguese versions of the MFQ scales. Table 1 shows the steps that were followed in the translation and adaptation process of the MFQ-C with the respective results: original version, version 1 (first forward translation), version 2 (second forward translation), reconciliation, back-translation and final version. The MFQ-P was also translated and adapted, and the table with the steps and results is available as Online-Only Supplementary Material.

Table 1 - Summary of results of translation and adaptation of MFQ into Brazilian Portuguese

\begin{tabular}{|c|c|c|c|c|c|}
\hline Original & Version 1 (V1) & Version 2 (V2) & Reconciliation (V3) & Back translation & Final version \\
\hline $\begin{array}{l}\text { This form is about } \\
\text { how you might have } \\
\text { been feeling or } \\
\text { acting recently. }\end{array}$ & $\begin{array}{l}\text { Este questionário } \\
\text { refere-se à forma } \\
\text { que você vem } \\
\text { se sentindo ou } \\
\text { se comportando } \\
\text { recentemente. }\end{array}$ & $\begin{array}{l}\text { Esse formulário } \\
\text { trata a respeito } \\
\text { de como você } \\
\text { pode estar agindo } \\
\text { ou se sentindo } \\
\text { recentemente. }\end{array}$ & $\begin{array}{l}\text { Este questionário } \\
\text { é sobre como você } \\
\text { pode estar se } \\
\text { sentindo ou agindo } \\
\text { recentemente. }\end{array}$ & $\begin{array}{l}\text { This questionnaire } \\
\text { is about how you } \\
\text { have been feeling or } \\
\text { acting recently. }\end{array}$ & $\begin{array}{l}\text { Este questionário } \\
\text { é sobre como você } \\
\text { pode estar se } \\
\text { sentindo ou agindo } \\
\text { recentemente. }\end{array}$ \\
\hline $\begin{array}{l}\text { For each question, } \\
\text { please check }(\checkmark) \\
\text { how you have been } \\
\text { feeling or acting } \\
\text { in the past two } \\
\text { weeks. }\end{array}$ & $\begin{array}{l}\text { Para cada } \\
\text { pergunta, por } \\
\text { favor marque }(\checkmark) \\
\text { como você vem } \\
\text { se sentindo ou } \\
\text { se comportando } \\
\text { nas últimas duas } \\
\text { semanas. }\end{array}$ & $\begin{array}{l}\text { Para cada pergunta, } \\
\text { marque (v) como } \\
\text { você tem se sentido } \\
\text { ou agido nas últimas } \\
\text { duas semanas. }\end{array}$ & $\begin{array}{l}\text { Para cada pergunta, } \\
\text { por favor marque }(\checkmark) \\
\text { como você esteve se } \\
\text { sentindo ou agindo } \\
\text { nas últimas duas } \\
\text { semanas. }\end{array}$ & $\begin{array}{l}\text { For each question, } \\
\text { check }(\checkmark) \text { how you } \\
\text { have been feeling or } \\
\text { acting over the past } \\
\text { two weeks. }\end{array}$ & $\begin{array}{l}\text { Para cada pergunta, } \\
\text { por favor marque } \\
(\checkmark) \text { como você } \\
\text { esteve se sentindo } \\
\text { ou agindo nas } \\
\text { últimas duas } \\
\text { semanas. }\end{array}$ \\
\hline $\begin{array}{l}\text { If a sentence was } \\
\text { not true about you, } \\
\text { check NOT TRUE. } \\
\text { If a sentence was } \\
\text { only sometimes true, } \\
\text { check SOMETIMES. } \\
\text { If a sentence was } \\
\text { true about you most } \\
\text { of the time, check } \\
\text { TRUE }\end{array}$ & $\begin{array}{l}\text { Se a frase não é } \\
\text { verdadeira sobre } \\
\text { você, marque NÃ̃ } \\
\text { É VERDADEIRA. } \\
\text { Se a frase é } \\
\text { apenas às vezes } \\
\text { verdadeira, marque } \\
\text { ÀS VEZES. } \\
\text { Se a frase é } \\
\text { verdadeira sobre } \\
\text { você na maioria } \\
\text { do tempo, marque } \\
\text { VERDADEIRA. }\end{array}$ & $\begin{array}{l}\text { Se uma frase não } \\
\text { condiz com você, } \\
\text { marque Não é } \\
\text { Verdade } \\
\text { Se uma frase condiz } \\
\text { com você somente } \\
\text { às vezes, marque } \\
\text { Ocasionalmente } \\
\text { Verdade } \\
\text { Se uma frase condiz } \\
\text { com você na maior } \\
\text { parte do tempo, } \\
\text { marque Verdade }\end{array}$ & $\begin{array}{l}\text { Se a frase não for } \\
\text { verdadeira sobre } \\
\text { você, marque NÃO É } \\
\text { VERDADE. } \\
\text { Se a frase for } \\
\text { verdadeira apenas } \\
\text { às vezes, marque ÀS } \\
\text { VEZES. } \\
\text { Se a frase for } \\
\text { verdadeira sobre } \\
\text { você na maior parte } \\
\text { do tempo, marque } \\
\text { VERDADE }\end{array}$ & $\begin{array}{l}\text { If the phrase is not } \\
\text { true about you, } \\
\text { mark NOT TRUE. } \\
\text { If the phrase is only } \\
\text { true sometimes, } \\
\text { mark SOMETIMES. } \\
\text { If the phrase is true } \\
\text { about you most } \\
\text { of the time, mark } \\
\text { TRUE. }\end{array}$ & $\begin{array}{l}\text { Se a frase não for } \\
\text { verdadeira sobre } \\
\text { você, marque NÃO É } \\
\text { VERDADE. } \\
\text { Se a frase for } \\
\text { verdadeira apenas } \\
\text { às vezes, marque ÀS } \\
\text { VEZES. } \\
\text { Se a frase for } \\
\text { verdadeira sobre } \\
\text { você na maior parte } \\
\text { do tempo, marque } \\
\text { VERDADE. }\end{array}$ \\
\hline $\begin{array}{l}\text { Score the MFQ as } \\
\text { follows: } \\
\text { NOT TRUE }=0 \\
\text { SOMETIMES = } 1 \\
\text { TRUE }=2\end{array}$ & $\begin{array}{l}\text { Pontue o MFQ da } \\
\text { seguinte forma: } \\
\text { NÃO É } \\
\text { VERDADEIRA = } 0 \\
\text { ÀS VEZES }=1 \\
\text { VERDADEIRA = } 2\end{array}$ & $\begin{array}{l}\text { Pontue o QHS da } \\
\text { seguinte forma: } \\
\text { Não é verdade }=0 \\
\text { Ocasionalmente } \\
\text { Verdade }=1 \\
\text { Verdade }=2\end{array}$ & $\begin{array}{l}\text { Pontue o MFQ da } \\
\text { seguinte forma: } \\
\text { NẤ É VERDADE }=0 \\
\text { ÀS VEZES }=1 \\
\text { VERDADE }=2\end{array}$ & $\begin{array}{l}\text { Scoring: } \\
\text { NOT TRUE }=0 \\
\text { SOMETIMES }=1 \\
\text { TRUE }=2\end{array}$ & $\begin{array}{l}\text { Pontue o MFQ da } \\
\text { seguinte forma: } \\
\text { NẤ É VERDADE }=0 \\
\text { ÀS VEZES }=1 \\
\text { VERDADE }=2\end{array}$ \\
\hline $\begin{array}{l}\text { To code, please } \\
\text { use a checkmark } \\
(\checkmark) \text { for each } \\
\text { statement. }\end{array}$ & $\begin{array}{l}\text { Para preencher, } \\
\text { por favor, use o } \\
\text { sinal de " } \checkmark \text { " para } \\
\text { cada afirmação. }\end{array}$ & $\begin{array}{l}\text { Para preencher, } \\
\text { por favor, use o } \\
\text { sinal de " } \checkmark \text { " para } \\
\text { cada afirmação. }\end{array}$ & $\begin{array}{l}\text { Para preencher, por } \\
\text { favor, use o sinal } \\
\text { de " } \checkmark \text { " para cada } \\
\text { afirmação. }\end{array}$ & $\begin{array}{l}\text { To code, please } \\
\text { use a checkmark } \\
(\checkmark) \text { for each } \\
\text { statement. }\end{array}$ & $\begin{array}{l}\text { Para preencher, } \\
\text { por favor use o } \\
\text { sinal } \checkmark \text { para cada } \\
\text { afirmação. }\end{array}$ \\
\hline NOT TRUE & $\begin{array}{l}\text { NÃO É } \\
\text { VERDADEIRA }\end{array}$ & $\begin{array}{l}\text { NÃO É } \\
\text { VERDADEIRA }\end{array}$ & $\begin{array}{l}\text { NÃO É } \\
\text { VERDADEIRA }\end{array}$ & NOT TRUE & NÃO É VERDADE \\
\hline SOMETIMES & ÀS VEZES & ÀS VEZES & Às VEZES & SOMETIMES & ÀS VEZES \\
\hline TRUE & VERDADEIRA & VERDADEIRA & VERDADEIRA & TRUE & VERDADE \\
\hline
\end{tabular}




\begin{tabular}{|c|c|c|c|c|c|}
\hline Original & Version 1 (V1) & Version 2 (V2) & Reconciliation (V3) & Back translation & Final version \\
\hline $\begin{array}{l}\text { I felt miserable or } \\
\text { unhappy. }\end{array}$ & $\begin{array}{l}\text { Eu me senti muito } \\
\text { triste ou infeliz. }\end{array}$ & $\begin{array}{l}\text { Eu me sentia muito } \\
\text { triste ou infeliz. }\end{array}$ & $\begin{array}{l}\text { Eu me senti muito } \\
\text { triste ou infeliz. }\end{array}$ & $\begin{array}{l}\text { I have felt very sad } \\
\text { or unhappy. }\end{array}$ & $\begin{array}{l}\text { Eu me senti muito } \\
\text { triste ou infeliz. }\end{array}$ \\
\hline $\begin{array}{l}\text { I didn't enjoy } \\
\text { anything at all. }\end{array}$ & $\begin{array}{l}\text { Eu não consegui } \\
\text { sentir prazer em } \\
\text { nada. }\end{array}$ & $\begin{array}{l}\text { Eu não conseguia } \\
\text { apreciar/desfrutar de } \\
\text { nada. }\end{array}$ & $\begin{array}{l}\text { Eu não consegui me } \\
\text { divertir com nada. }\end{array}$ & $\begin{array}{l}\text { I haven't been able } \\
\text { to enjoy anything. }\end{array}$ & $\begin{array}{l}\text { Eu não consegui } \\
\text { me divertir com } \\
\text { absolutamente nada. }\end{array}$ \\
\hline $\begin{array}{l}\text { I was less hungry } \\
\text { than usual. }\end{array}$ & $\begin{array}{l}\text { Eu estava com } \\
\text { menos fome do } \\
\text { que o usual. }\end{array}$ & $\begin{array}{l}\text { Eu estava com } \\
\text { menos fome do que } \\
\text { usualmente. }\end{array}$ & $\begin{array}{l}\text { Eu estive com } \\
\text { menos fome do que } \\
\text { normalmente. }\end{array}$ & $\begin{array}{l}\text { I have been less } \\
\text { hungry than usual. }\end{array}$ & $\begin{array}{l}\text { Eu estive com } \\
\text { menos fome do que } \\
\text { normalmente. }\end{array}$ \\
\hline $\begin{array}{l}\text { I ate more than } \\
\text { usual. }\end{array}$ & $\begin{array}{l}\text { Eu comi mais do } \\
\text { que o usual. }\end{array}$ & $\begin{array}{l}\text { Eu comia mais do } \\
\text { que usualmente. }\end{array}$ & $\begin{array}{l}\text { Eu comi mais do que } \\
\text { normalmente. }\end{array}$ & $\begin{array}{l}\text { I have been eating } \\
\text { more than usual. }\end{array}$ & $\begin{array}{l}\text { Eu comi mais do que } \\
\text { normalmente. }\end{array}$ \\
\hline $\begin{array}{l}\text { I felt so tired I just } \\
\text { sat around and did } \\
\text { nothing. }\end{array}$ & $\begin{array}{l}\text { Eu me senti tão } \\
\text { cansado (a) que } \\
\text { apenas fiquei } \\
\text { sentado (a) sem } \\
\text { fazer nada. }\end{array}$ & $\begin{array}{l}\text { Eu me sentia tão } \\
\text { cansado que só } \\
\text { ficava sentado e não } \\
\text { fazia nada. }\end{array}$ & $\begin{array}{l}\text { Eu me senti tão } \\
\text { cansado(a) que } \\
\text { apenas ficava } \\
\text { sentado(a) sem fazer } \\
\text { nada. }\end{array}$ & $\begin{array}{l}\text { I have felt so tired } \\
\text { all I can do is sit and } \\
\text { do nothing. }\end{array}$ & $\begin{array}{l}\text { Eu me senti tão } \\
\text { cansado(a) que só } \\
\text { ficava sentado(a) } \\
\text { sem fazer nada. }\end{array}$ \\
\hline $\begin{array}{l}\text { I was moving and } \\
\text { walking more slowly } \\
\text { than usual. }\end{array}$ & $\begin{array}{l}\text { Eu estava me } \\
\text { movimentando e } \\
\text { caminhando mais } \\
\text { devagar do que o } \\
\text { usual. }\end{array}$ & $\begin{array}{l}\text { Eu estava me } \\
\text { mexendo e } \\
\text { caminhando mais } \\
\text { lentamente que o } \\
\text { usual. }\end{array}$ & $\begin{array}{l}\text { Eu estive me } \\
\text { movimentando e } \\
\text { caminhando mais } \\
\text { devagar do que } \\
\text { normalmente. }\end{array}$ & $\begin{array}{l}\text { I have been moving } \\
\text { and walking more } \\
\text { slowly than usual. }\end{array}$ & $\begin{array}{l}\text { Eu estive me } \\
\text { movimentando e } \\
\text { caminhando mais } \\
\text { devagar do que } \\
\text { normalmente. }\end{array}$ \\
\hline I was very restless. & $\begin{array}{l}\text { Eu estava muito } \\
\text { inquieto (a). }\end{array}$ & $\begin{array}{l}\text { Eu me sentia muito } \\
\text { inquieto/agitado. }\end{array}$ & $\begin{array}{l}\text { Eu estive muito } \\
\text { agitado(a). }\end{array}$ & $\begin{array}{l}\text { I have felt really } \\
\text { restless. }\end{array}$ & $\begin{array}{l}\text { Eu estive muito } \\
\text { agitado(a). }\end{array}$ \\
\hline $\begin{array}{l}\text { I felt I was no good } \\
\text { anymore. }\end{array}$ & $\begin{array}{l}\text { Eu senti que } \\
\text { não tinha mais } \\
\text { importância. }\end{array}$ & $\begin{array}{l}\text { Eu sentia que não } \\
\text { era mais uma } \\
\text { pessoa boa. }\end{array}$ & $\begin{array}{l}\text { Eu senti que eu não } \\
\text { prestava mais. }\end{array}$ & I feel worthless. & $\begin{array}{l}\text { Eu senti que eu não } \\
\text { valia mais nada. }\end{array}$ \\
\hline $\begin{array}{l}\text { I blamed myself for } \\
\text { things that weren't } \\
\text { my fault. }\end{array}$ & $\begin{array}{l}\text { Eu me culpava por } \\
\text { coisas que não } \\
\text { eram minha culpa. }\end{array}$ & $\begin{array}{l}\text { Eu me culpava por } \\
\text { coisas pelas quais } \\
\text { não tinha culpa. }\end{array}$ & $\begin{array}{l}\text { Eu me culpei por } \\
\text { coisas que não eram } \\
\text { minha culpa. }\end{array}$ & $\begin{array}{l}\text { I have blamed } \\
\text { myself for things } \\
\text { that aren't my fault. }\end{array}$ & $\begin{array}{l}\text { Eu me culpei por } \\
\text { coisas que não eram } \\
\text { minha culpa. }\end{array}$ \\
\hline $\begin{array}{l}\text { It was hard for me } \\
\text { to make up my } \\
\text { mind. }\end{array}$ & $\begin{array}{l}\text { Era difícil para mim } \\
\text { tomar decisões. }\end{array}$ & $\begin{array}{l}\text { Era difícil para mim } \\
\text { tomar uma decisão. }\end{array}$ & $\begin{array}{l}\text { Foi difícil me decidir } \\
\text { sobre as coisas. }\end{array}$ & $\begin{array}{l}\text { I have had trouble } \\
\text { making decisions. }\end{array}$ & $\begin{array}{l}\text { Foi difícil me decidir } \\
\text { sobre as coisas. }\end{array}$ \\
\hline $\begin{array}{l}\text { I felt grumpy and } \\
\text { cross with my } \\
\text { parents. }\end{array}$ & $\begin{array}{l}\text { Eu me senti mal- } \\
\text { humorado }(a) \text { e } \\
\text { irritado }(a) \text { com } \\
\text { meus pais. }\end{array}$ & $\begin{array}{l}\text { Eu me sentia mal- } \\
\text { humorado e irritado } \\
\text { com meus pais. }\end{array}$ & $\begin{array}{l}\text { Eu fiquei } \\
\text { emburrado(a) e de } \\
\text { mal com meus pais. }\end{array}$ & $\begin{array}{l}\text { I have been irritated } \\
\text { and crabby with my } \\
\text { parents. }\end{array}$ & $\begin{array}{l}\text { Eu fiquei } \\
\text { emburrado(a) e de } \\
\text { mal com meus pais. }\end{array}$ \\
\hline $\begin{array}{l}\text { I felt like talking less } \\
\text { than usual. }\end{array}$ & $\begin{array}{l}\text { Eu senti que eu } \\
\text { estava menos } \\
\text { disposto (a) a } \\
\text { conversar do que o } \\
\text { usual. }\end{array}$ & $\begin{array}{l}\text { Eu me sentia menos } \\
\text { a fim de conversar } \\
\text { do que o usual. }\end{array}$ & $\begin{array}{l}\text { Eu estive menos a fim } \\
\text { de conversar do que } \\
\text { normalmente. }\end{array}$ & $\begin{array}{l}\text { I've been less } \\
\text { talkative than usual. }\end{array}$ & $\begin{array}{l}\text { Eu estive menos a } \\
\text { fim de conversar do } \\
\text { que normalmente. }\end{array}$ \\
\hline $\begin{array}{l}\text { I was talking more } \\
\text { slowly than usual. }\end{array}$ & $\begin{array}{l}\text { Eu estava falando } \\
\text { mais devagar do } \\
\text { que o usual. }\end{array}$ & $\begin{array}{l}\text { Eu sentia que } \\
\text { estava falando mais } \\
\text { lentamente que o } \\
\text { usual. }\end{array}$ & $\begin{array}{l}\text { Minha fala esteve } \\
\text { mais devagar do que } \\
\text { normalmente. }\end{array}$ & $\begin{array}{l}\text { I have been talking } \\
\text { more slowly than } \\
\text { usual. }\end{array}$ & $\begin{array}{l}\text { Minha fala esteve } \\
\text { mais devagar do que } \\
\text { normalmente. }\end{array}$ \\
\hline I cried a lot. & Eu chorava muito. & Eu chorava muito. & Eu chorei muito. & $\begin{array}{l}\text { I have been crying } \\
\text { a lot. }\end{array}$ & Eu chorei muito. \\
\hline $\begin{array}{l}\text { I thought there was } \\
\text { nothing good for me } \\
\text { in the future. }\end{array}$ & $\begin{array}{l}\text { Eu pensava que } \\
\text { nada de bom } \\
\text { aconteceria comigo } \\
\text { no futuro. }\end{array}$ & $\begin{array}{l}\text { Eu pensava que não } \\
\text { havia nada de bom } \\
\text { para mim no futuro. }\end{array}$ & $\begin{array}{l}\text { Eu pensei que nada } \\
\text { de bom aconteceria } \\
\text { comigo no futuro. }\end{array}$ & $\begin{array}{l}\text { I think nothing good } \\
\text { is ever going to } \\
\text { happen to me. }\end{array}$ & $\begin{array}{l}\text { Eu pensei que nada } \\
\text { de bom aconteceria } \\
\text { comigo no futuro. }\end{array}$ \\
\hline $\begin{array}{l}\text { I thought that life } \\
\text { wasn't worth living. }\end{array}$ & $\begin{array}{l}\text { Eu pensei que a } \\
\text { vida não valia a } \\
\text { pena ser vivida. }\end{array}$ & $\begin{array}{l}\text { Eu pensava que } \\
\text { a vida não valia a } \\
\text { pena ser vivida. }\end{array}$ & $\begin{array}{l}\text { Eu pensei que a vida } \\
\text { não valia a pena ser } \\
\text { vivida. }\end{array}$ & $\begin{array}{l}\text { I think life is not } \\
\text { worth living. }\end{array}$ & $\begin{array}{l}\text { Eu pensei que a vida } \\
\text { não valia a pena ser } \\
\text { vivida. }\end{array}$ \\
\hline
\end{tabular}




\begin{tabular}{|c|c|c|c|c|c|}
\hline Original & Version 1 (V1) & Version 2 (V2) & Reconciliation (V3) & Back translation & Final version \\
\hline $\begin{array}{l}\text { I thought about } \\
\text { death or dying. }\end{array}$ & $\begin{array}{l}\text { Eu pensei sobre } \\
\text { morte ou morrer. }\end{array}$ & $\begin{array}{l}\text { Eu pensava a } \\
\text { respeito da morte ou } \\
\text { de morrer }\end{array}$ & $\begin{array}{l}\text { Eu pensei sobre morte } \\
\text { ou morrer. }\end{array}$ & $\begin{array}{l}\text { I have thought about } \\
\text { death or dying. }\end{array}$ & $\begin{array}{l}\text { Eu pensei sobre } \\
\text { morte ou morrer. }\end{array}$ \\
\hline $\begin{array}{l}\text { I thought my family } \\
\text { would be better off } \\
\text { without me. }\end{array}$ & $\begin{array}{l}\text { Eu pensei que } \\
\text { a minha família } \\
\text { poderia ser mais } \\
\text { feliz sem a minha } \\
\text { presença. }\end{array}$ & $\begin{array}{l}\text { Eu pensava que } \\
\text { minha família estaria } \\
\text { melhor sem mim. }\end{array}$ & $\begin{array}{l}\text { Eu pensei que minha } \\
\text { família estaria melhor } \\
\text { sem mim. }\end{array}$ & $\begin{array}{l}\text { I have thought that } \\
\text { my family would be } \\
\text { better off without } \\
\text { me. }\end{array}$ & $\begin{array}{l}\text { Eu pensei que minha } \\
\text { família estaria } \\
\text { melhor sem mim. }\end{array}$ \\
\hline $\begin{array}{l}\text { I thought about } \\
\text { killing myself. }\end{array}$ & $\begin{array}{l}\text { Eu pensei em me } \\
\text { matar. }\end{array}$ & $\begin{array}{l}\text { Eu pensava em me } \\
\text { matar. }\end{array}$ & $\begin{array}{l}\text { Eu pensei em me } \\
\text { matar. }\end{array}$ & $\begin{array}{l}\text { I've thought about } \\
\text { killing myself. }\end{array}$ & $\begin{array}{l}\text { Eu pensei em me } \\
\text { matar. }\end{array}$ \\
\hline $\begin{array}{l}\text { I didn't want to see } \\
\text { my friends. }\end{array}$ & $\begin{array}{l}\text { Eu não queria ver } \\
\text { meus amigos. }\end{array}$ & $\begin{array}{l}\text { Eu não queria ver } \\
\text { meus amigos. }\end{array}$ & $\begin{array}{l}\text { Eu não queria ver } \\
\text { meus amigos. }\end{array}$ & $\begin{array}{l}\text { I haven't wanted to } \\
\text { see my friends. }\end{array}$ & $\begin{array}{l}\text { Eu não queria ver } \\
\text { meus amigos. }\end{array}$ \\
\hline $\begin{array}{l}\text { I found it hard to } \\
\text { think properly or } \\
\text { concentrate. }\end{array}$ & $\begin{array}{l}\text { Eu achei } \\
\text { difícil pensar } \\
\text { corretamente ou } \\
\text { me concentrar. }\end{array}$ & $\begin{array}{l}\text { Eu achava difícil } \\
\text { pensar corretamente } \\
\text { e me concentrar. }\end{array}$ & $\begin{array}{l}\text { Eu achei difícil } \\
\text { raciocinar ou me } \\
\text { concentrar. }\end{array}$ & $\begin{array}{l}\text { I have had trouble } \\
\text { thinking or } \\
\text { concentrating. }\end{array}$ & $\begin{array}{l}\text { Eu achei difícil } \\
\text { raciocinar ou me } \\
\text { concentrar. }\end{array}$ \\
\hline $\begin{array}{l}\text { I thought bad things } \\
\text { would happen to me. }\end{array}$ & $\begin{array}{l}\text { Eu pensei que } \\
\text { coisas ruins } \\
\text { aconteceriam } \\
\text { comigo. }\end{array}$ & $\begin{array}{l}\text { Eu achava que } \\
\text { coisas ruins iriam } \\
\text { acontecer comigo. }\end{array}$ & $\begin{array}{l}\text { Eu pensei que coisas } \\
\text { ruins aconteceriam } \\
\text { comigo. }\end{array}$ & $\begin{array}{l}\text { I think bad things } \\
\text { will happen to me. }\end{array}$ & $\begin{array}{l}\text { Eu pensei que coisas } \\
\text { ruins aconteceriam } \\
\text { comigo. }\end{array}$ \\
\hline I hated myself. & Eu me odiei. & Eu me odiava. & Eu me odiei. & I hate myself. & Eu me odiei. \\
\hline $\begin{array}{l}\text { I felt I was a bad } \\
\text { person. }\end{array}$ & $\begin{array}{l}\text { Eu me senti uma } \\
\text { pessoa ruim. }\end{array}$ & $\begin{array}{l}\text { Eu sentia que era } \\
\text { uma má pessoa. }\end{array}$ & $\begin{array}{l}\text { Eu me senti uma } \\
\text { pessoa ruim. }\end{array}$ & $\begin{array}{l}\text { I feel like a bad } \\
\text { person. }\end{array}$ & $\begin{array}{l}\text { Eu me senti uma } \\
\text { pessoa ruim. }\end{array}$ \\
\hline $\begin{array}{l}\text { I thought I looked } \\
\text { ugly. }\end{array}$ & $\begin{array}{l}\text { Eu pensei que era } \\
\text { feio }(a) \text {. }\end{array}$ & $\begin{array}{l}\text { Eu pensava que era } \\
\text { feio. }\end{array}$ & Eu me senti feio(a). & I feel ugly. & Eu me senti feio(a). \\
\hline $\begin{array}{l}\text { I worried about } \\
\text { aches and pains. }\end{array}$ & $\begin{array}{l}\text { Eu me preocupei } \\
\text { com dores e } \\
\text { sofrimentos. }\end{array}$ & $\begin{array}{l}\text { Eu me preocupava } \\
\text { com dores. }\end{array}$ & $\begin{array}{l}\text { Eu me preocupei com } \\
\text { dores no corpo. }\end{array}$ & $\begin{array}{l}\text { I am worried about } \\
\text { pain in my body. }\end{array}$ & $\begin{array}{l}\text { Eu me preocupei } \\
\text { com dores no corpo. }\end{array}$ \\
\hline I felt lonely. & $\begin{array}{l}\text { Eu me senti } \\
\text { sozinho }(a) \text {. }\end{array}$ & $\begin{array}{l}\text { Eu me sentia } \\
\text { sozinho. }\end{array}$ & $\begin{array}{l}\text { Eu me senti } \\
\text { sozinho(a). }\end{array}$ & I have felt alone. & $\begin{array}{l}\text { Eu me senti } \\
\text { sozinho(a). }\end{array}$ \\
\hline $\begin{array}{l}\text { I thought nobody } \\
\text { really loved me. }\end{array}$ & $\begin{array}{l}\text { Eu pensei que } \\
\text { ninguém poderia } \\
\text { realmente gostar } \\
\text { de mim. }\end{array}$ & $\begin{array}{l}\text { Eu acreditava que } \\
\text { ninguém realmente } \\
\text { me amava. }\end{array}$ & $\begin{array}{l}\text { Eu pensei que } \\
\text { ninguém me amava } \\
\text { de verdade. }\end{array}$ & $\begin{array}{l}\text { I feel like no one } \\
\text { really loves me. }\end{array}$ & $\begin{array}{l}\text { Eu pensei que } \\
\text { ninguém me amava } \\
\text { de verdade. }\end{array}$ \\
\hline $\begin{array}{l}\text { I didn't have any fun } \\
\text { in school. }\end{array}$ & $\begin{array}{l}\text { Eu não me divertia } \\
\text { na escola. }\end{array}$ & $\begin{array}{l}\text { Eu não me divertia } \\
\text { em nada na escola. }\end{array}$ & $\begin{array}{l}\text { Eu não me diverti nem } \\
\text { um pouco nas minhas } \\
\text { atividades. }\end{array}$ & $\begin{array}{l}\text { I haven't had any } \\
\text { fun with my usual } \\
\text { activities. }\end{array}$ & $\begin{array}{l}\text { Eu não me diverti } \\
\text { nem um pouco nas } \\
\text { minhas atividades. }\end{array}$ \\
\hline $\begin{array}{l}\text { I thought I could } \\
\text { never be as good as } \\
\text { other kids. }\end{array}$ & $\begin{array}{l}\text { Eu pensei que eu } \\
\text { jamais poderia } \\
\text { ser bom como as } \\
\text { outras crianças. }\end{array}$ & $\begin{array}{l}\text { Eu achava que } \\
\text { nunca seria tão bom } \\
\text { quanto as outras } \\
\text { crianças. }\end{array}$ & $\begin{array}{l}\text { Eu pensei que eu } \\
\text { nunca seria tão } \\
\text { bom(boa) quanto } \\
\text { os outros da minha } \\
\text { idade. }\end{array}$ & $\begin{array}{l}\text { I don't think I'll ever } \\
\text { be as good as other } \\
\text { kids my age. }\end{array}$ & $\begin{array}{l}\text { Eu pensei que eu } \\
\text { nunca seria tão } \\
\text { bom(boa) quanto } \\
\text { os outros da minha } \\
\text { idade. }\end{array}$ \\
\hline $\begin{array}{l}\text { I did everything } \\
\text { wrong. }\end{array}$ & $\begin{array}{l}\text { Eu fazia tudo } \\
\text { errado. }\end{array}$ & Eu fazia tudo errado. & Eu fiz tudo errado. & $\begin{array}{l}\text { I do everything } \\
\text { wrong. }\end{array}$ & Eu fiz tudo errado. \\
\hline $\begin{array}{l}\text { I didn't sleep as well } \\
\text { as I usually sleep. }\end{array}$ & $\begin{array}{l}\text { Eu não dormi tão } \\
\text { bem como eu } \\
\text { usualmente durmo. }\end{array}$ & $\begin{array}{l}\text { Eu não dormia tão } \\
\text { bem quanto durmo } \\
\text { usualmente. }\end{array}$ & $\begin{array}{l}\text { Eu não dormi tão } \\
\text { bem quanto eu } \\
\text { normalmente durmo. }\end{array}$ & $\begin{array}{l}\text { I haven't been } \\
\text { sleeping as well as } \\
\text { usual. }\end{array}$ & $\begin{array}{l}\text { Eu não dormi tão } \\
\text { bem quanto eu } \\
\text { normalmente durmo. }\end{array}$ \\
\hline $\begin{array}{l}\text { I slept a lot more } \\
\text { than usual. }\end{array}$ & $\begin{array}{l}\text { Eu dormi mais do } \\
\text { que o usual. }\end{array}$ & $\begin{array}{l}\text { Eu dormia } \\
\text { muito mais que } \\
\text { usualmente. }\end{array}$ & $\begin{array}{l}\text { Eu dormi muito mais } \\
\text { do que normalmente. }\end{array}$ & $\begin{array}{l}\text { I have been sleeping } \\
\text { much more than } \\
\text { usual. }\end{array}$ & $\begin{array}{l}\text { Eu dormi muito } \\
\text { mais do que } \\
\text { normalmente. }\end{array}$ \\
\hline
\end{tabular}


Following the steps in Table 1 , between backtranslation and final version, two versions of the instrument (MFQ-C and MFQ-P) were administered to 28 adolescents and primary caregivers whose native language was Brazilian Portuguese. For each item, patients answered the following questions: "Do you understand this instruction/item/rating scale? (If you do not understand, please, explain the difficulty)"; "If there are difficulties, how would you write this instruction/item/rating scale?"; "Could you explain what it means? (Ask the person to explain what they think that the instruction/item/rating scale means)"; "Are the response options appropriate? (If they aren't, please explain the difficulty and how you would write it)." Adolescents and caregivers took an average of 30 to 45 minutes to respond to the questionnaire. All responses were assessed in detail by a decision committee, which consensually decided that no additional changes were required, as both MFQ versions were shown to be easily understood. The only issue that raised some questions by the adolescents was a possible overlap between the item on morbid thoughts (item 17 - "Eu pensei sobre morte ou morrer") and the one on suicidal ideation (item 19 - "Eu pensei em me matar").

\section{Discussion}

The MFQ is one of the most frequently used instruments to assess depressive symptoms in children and adolescents in the international literature. ${ }^{12,14}$ The absence of a Brazilian Portuguese translation of its long version prevents its use in a country currently with a population of 63 million children and adolescents. ${ }^{15} \mathrm{We}$ have described the process of translation and cultural adaptation of the MFQ using the methods recommended by the ISPOR. Both MFQ-C and MFQ-P, after rigorous procedures of translation and back-translation, were tested in a sample of adolescent patients and caregivers, who verified the comprehensibility of the scale. The Brazilian Portuguese translation of the long versions of the MFQ appeared to be acceptable and easy to understand.

Other instruments that evaluate the symptoms of MDD in this age group have also been translated and validated for Brazilian Portuguese. The CDI was initially translated and validated in $1995^{16}$ and, since then, it has been applied with the objective of verifying its psychometric properties. ${ }^{17,18}$ The BDI was also translated and validated to Brazilian Portuguese, ${ }^{19}$ and several studies have evaluated the psychometric properties of this instrument among Brazilian adolescents. ${ }^{20,21}$
The CESD has also been translated and validated into Brazilian Portuguese, and its psychometric properties have been verified in different populations, including adolescents. ${ }^{22-27}$

Collecting information from multiple sources is essential in the assessment of psychiatric symptoms among children and adolescents. ${ }^{28}$ As the MFQ also includes a version for parents, this allows the instrument to be even more informative. Although MDD is conceptualized as an internalizing disorder, the caregiver's version helps to capture the construct of depression more comprehensively and seems to better predict a depressive episode when compared to the adolescent report in isolation. ${ }^{8,14}$

The present study, however, is not without limitations: the sample for the cognitive debriefing step was very small and, despite trying to cover different ages and genders, we did not control for socioeconomic status or diagnostic categories. Also, the fact that all subjects were patients at mental health clinics limits the generalizability of findings to community settings. Additionally, although we also translated the MFQ-A, we did not include it in the cognitive debriefing for logistical reasons. Nevertheless, the MFQ-A is extremely similar to both MFQ-C and MFQ-P, which suggests that adults would not have difficulty understanding it.

Translation and cultural adaptation constitute a first step in the process of validation of an instrument in a different setting. The Brazilian Portuguese versions of the MFQ are now ready for use with adolescents and caregivers. Future studies should evaluate the psychometric properties of the questionnaires in order to gain insights into the reliability and validity of the instrument in a more diverse population.

\section{Acknowledgments}

Christian Kieling received grants from Conselho Nacional de Desenvolvimento Científico e Tecnológico (CNPq), Coordenação de Aperfeiçoamento de Pessoal de Nível Superior (CAPES), Fundação de Amparo à Pesquisa do Estado do Rio Grande do Sul (FAPERGS), and Fundo de Incentivo a Pesquisa e Eventos - Hospital de Clínicas de Porto Alegre (FIPE/HCPA).

The authors are grateful to Brian Small and Jane Costello for the authorization to translate the questionnaires and for the revision of the back-translated versions of MFQ. The authors also thank Cristiane Geyer, Dimas Gramz, Julia Bondar and Vinícius Martins-Costa for their assistance in the process of cognitive debriefing. 


\section{References}

1. Whiteford $H A$, Degenhardt $L$, Rehm J, Baxter $A]$, Ferrari $A J$ Erskine $\mathrm{HE}$, et al. Global burden of disease attributable to mental and substance use disorders: findings from the Global Burden of Disease Study 2010. Lancet. 2013;382:1575-86.

2. Rocha TBM, Kieling C, Graeff-Martins AS, Rohde LA. Provision of mental healthcare for children and adolescents. Curr Opin Psychiatry. 2015;28:330-5.

3. Stockings $E$, Degenhardt L, Lee YY, Mihalopoulos C, Liu A, Hobbs $M$, et al. Symptom screening scales for detecting major depressive disorder in children and adolescents: a systematic review and meta-analysis of reliability, validity and diagnostic utility. J Affect Disord. 2015;11:341-76.

4. Beck AT, Ward CH, Mendelson M, Mock J, Erbaugh J. An inventory for measuring depression. Arch Gen Psychiatry. 1961;4:561-71.

5. Radloff LS. The CES-D scale: a self-report depression scale for research in the general population. Appl Psychol Meas. 1977;1:385-401.

6. Munguía JR, Ochoa $\mathrm{H}$, Casarín A, Palos P. Evaluation of differential item functioning of the Center of Epidemiological Scale Depression Revisited for gender in Mexican Adolescents. Psychol Res. 2013;3232-41.

7. Kovacs M. The Children's Depression Inventory (CDI). Psychopharmacol Bull. 1984;21:995-8.

8. Daviss WB, Birmaher B, Melhem NA, Axelson DA, Michaels SM, Brent DA. Criterion validity of the Mood and Feelings Questionnaire for depressive episodes in clinic and non-clinic subjects. J Child Psychol Psychiatry. 2006;47:927-34.

9. Costello EJ, Angold A. Scales to assess child and adolescent depression: checklists, screens, and nets. J Am Acad Child Adolesc Psychiatry. 1988;27:726-37.

10. Angold A, Costello EJ, Messer SC, Pickles A. Development of a short questionnaire for use in epidemiological studies of depression in children and adolescents. Int J Methods Psychiatr Res. 1995; 5:237-49.

11. Pinto ERS. Short Mood and Feelings Questionnaire: tradução para língua portuguesa, adaptação cultural e validação [dissertation]. São Paulo: USP; 2014.

12. Goodyer I, Reynolds S, Barrett B, Byford S, Dubicka B, Hill J, et al. Cognitive behavioural therapy and short-term psychoanalytical psychotherapy versus a brief psychosocial intervention in adolescents with unipolar major depressive disorder (IMPACT): a multicentre, pragmatic, observer-blind, randomised controlled superiority trial. Lancet. 2016;4:109-19.

13. Wild D, Grove A, Martin M, Eremenco S, McElroy S, VerjeeLorenz $A$, et al. ISPOR Task Force for Translation and Cultural Adaptation. Principles of good practice for the translation and cultural adaptation process for patient-reported outcomes (PRO) measures: report of the ISPOR Task Force for translation and cultural adaptation. Value Health. 2005;8:94-104.

14. Jeffreys M, Rozenman M, Gonzalez A, Warnick EM, Dauser C, Scahill $L$, et al. Factor Structure of the Parent-Report Mood and
Feelings Questionnaire (MFQ) in an outpatient mental health sample. J Abnorm Child Psychol. 2015;44:1111-20.

15. Instituto Brasileiro de Geografia e Estatística. Censo demográfico [Internet]. 2010. [cited 2016 Jan 05]. https:// www.ibge.gov.br

16. Gouveia V, Barbosa $G$, Almeida $H$, Gaião A. Inventário de Depressão Infantil - CDI: Estudo de adaptação com escolares de João Pessoa. J Bras Psiquiatr. 1995;44:345-9.

17. Wathier J, Lieberknecht J, Dell'Aglio DD, Bandeira DR. Análise fatorial do Inventário de Depressão Infantil (CDI) em amostra de jovens brasileiros. Aval Psicol. 2008;7:75-84.

18. Coutinho MP, Carolino ZS, Medeiros ED. Inventário de Depressão Infantil (CDI): evidências de validade de constructo e consistência interna. Aval Psicol. 2008;7:291-300.

19. Gorenstein C, Andrade L, Filho AHGV, Tung TC, Artes R. Psychometric properties of the Portuguese version of the Beck Depression Inventory on Brazilian college students. J Clin Psychol. 1999; 55:553-62.

20. Paranhos ME, Argimon II, Werlang BSG. Propriedades psicométricas do Inventário de Depressão de Beck-II (BDI-II) em adolescentes. Aval Psicol. 2010;9:383-92.

21. Argimon II, Terroso LB, Barbosa A, Lopes RM. Intensidade de sintomas depressivos em adolescentes através da escala de depressão de Beck (BDI-II). Bol Acad Paul Psicol. 2013;3385:35472.

22. Silveira DX, Jorge MR. Propriedades psicométricas da Escala de Rastreamento Populacional para Depressão CES-D em populações clínicas e não-clínicas de adolescentes e adultos jovens. Rev Psiquiatr Clin. 1998;25:251-61.

23. Schestatsky G. Desempenho de uma Escala de Rastreamento de Depressão (CES-D) em usuários de um serviço de cuidados primários de saúde de Porto Alegre [dissertation]. Porto Alegre: UFRGS; 2002.

24. Batistoni $S$, Neri AL, Cupertino AP. Validade da escala de depressão do Center for Epidemiological Studies (CES-D) entre idosos brasileiros. Rev Saude Publica. 2007;41:598-605.

25. Batistoni S, Neri AL, Cupertino AP. Validade e confiabilidade da versão brasileira da Center for Epidemiological Scale - Depression (CES-D) em estudantes brasileiros. Psico-USF. 2010;15:13-22.

26. Filho NH, Teixeira, M. A estrutura fatorial da Escala CES-D em estudantes universitários brasileiros. Aval Psicol. 2011;10:91-7.

27. Salle E, Rocha NS, Rocha TS, Nunes C, Chaves MLF. Escalas psicométricas como instrumentos de rastreamento para depressão em estudantes do ensino médio. Rev Psiquiatr Clin. 2012;39:247.

28. King RA. Practice parameters for the psychiatric assessment of children and adolescents. J Am Acad Child Adolesc Psychiatry. 1997;36:4-20

\section{Correspondence:}

Christian Kieling

Rua Ramiro Barcelos, 2350 - 400N

90035-903 - Porto Alegre, RS - Brazil

E-mail: ckieling@ufrgs.br 